\title{
Use of CAD Systems in the Training of Specialists in Analogue Circuitry
}

\author{
Valery K. Khokhlov ${ }^{1}$, Marina $V$. Gonobina ${ }^{1}$, Konstsntin P. Likhoedenko ${ }^{1}$, and Vitaliy V. \\ Glazkov $^{1 *}$ \\ ${ }^{1}$ Bauman Moscow State Technical University, 2nd Baumanskaya str., 5/1, 105005, Moscow, Russia
}

\begin{abstract}
Training of specialists in the field of analogue circuitry requires to study modern software that facilitates and accelerates the development of electronic devices. In this paper, we analyse the current CAD systems, which may be used in teaching analogue circuitry. We propose the approach of using CAD systems in all kinds of classroom activities. This approach is shown by the example of learning active analogue filters.
\end{abstract}

\section{Introduction}

There are several factors that should be considered in the curriculum development and choice of education technologies in the training of specialists in the field of analogue circuitry. These factors include having many prerequisites, such as microelectronics, electric circuit theory, theory of stability of technical systems, filter theory, operation calculus, etc. The lack of certain skills leads to having to study elements of these courses right in the analogue circuitry training process, which negatively impacts the quality of education.

Another important factor is that the object of study, the electronic components, are updated every year. Therefore, to be relevant on the labour market, it is necessary for the specialist to have competencies in the field of new circuit technology, methods of automated design of electronic equipment, and skills of working with the recent development tools.

Modern development tools allow to significantly increase the productivity of the developer of analogue electronic devices. For example, in [1] it is suggested to use MicroCAP CAD system for the synthesis of the finite impulse response filter using Fourier series method. In [2], the author suggests using this CAD system to model the operation of pulse and continuous converters of SEPIC architecture. The advantage of this approach is that the losses on the elements of the converters are taken into account, which allows to better estimate their efficiency. Overall, MicroCAP CAD system is a powerful tool for electronic devices modelling $[3,4]$.

It is shown in [5] that computer modelling for studying various types of active filters improves the training efficiency by increasing the motivation of students and their interest

* Corresponding author: glazkovv@bmstu.ru 
in the material. Therefore, even in the digital age, the interest in studying analogue circuitry remains high [6-13].

Because of that, many producers of analogue circuits provide various training materials designed for improving the efficiency of use of their products [14]. Moreover, the support consists not only of the provided technical descriptions and manuals, but also of the hardware platforms developed for carrying out experiments. Use of such tools in the learning process allows to substantially increase the labour market demand of the specialists in the field of analogue circuitry [15].

One of such examples is the ASLK PRO hardware platform, supplied with the educational materials, which describe the procedure of carrying out the experiments for studying analogue circuits [16-19]. More complex experiments can also be carried out using this hardware platform, including those containing the processing of mixed signals [20].

The limiting factor in the learning process is time, because a large amount of periodically changing material should be fit in the curriculum. Many calculations that before had to be done manually, now can be done using software tools $[3,4]$. It is more rational to use the time to study problem solving using these tools. Because of that, it is important to choose the most rational way of using CAD systems in the learning process.

\section{Types of CAD systems for solving analogue circuit modelling problems}

Currently we can distinguish the following types of CAD systems, which can be used in training of specialists in the field of analogue circuitry:

- CAD systems for end-to-end design of printed circuit boards. The examples of this kind of systems include Altium Designer, OrCAD, etc. The main concept of these tools is the support of full development cycle from simulation modelling of electronic devices to the development of circuit boards and modelling of signal integrity. Use of these tools in the early stages of learning analogue circuitry without having the knowledge of the electronic devices design basics is limited by the learning complexity. To start working with these tools, it is necessary to define the interaction with adjacent modules, which are not directly used in the modelling.

- CAD systems for electronic devices modelling, such as MicroCap, Proteus, etc. These systems have extensive libraries of electronic component models from leading world producers, are intuitive and allow special circuit analysis. For example, latest versions of MicroCap allow to model the subjective noise in the frequency domain and analyse the stability of circuits with negative feedback [3]. These tools are the most suitable for the early stages of training of specialists in the field of analogue circuitry.

- Utilities created by the chip producers. The leading world producers of analogue chips, such as Analog Devices, Texas Instruments, etc., provide the software of various complexity as a form of developer support, from Excel calculations to full modelling software, such as TINA-TI or LTspice. The advantage of these products is that they contain the information about electronic components straight from the chip producers. Usually they are less functional than the third-party CAD systems, however, they are more convenient when working with a specific chip. Moreover, there are utilities for solving a particular problem, such as FilterPro by Texas Instruments, which allows a digital filter synthesis based on operational amplifiers. 
The analysis shows that nowadays there is a wide range of CAD systems with various complexity and functionality. The choice of a software product should be made based on the problem. It is reasonable to use a CAD system for electronic system modelling as a main tool, and utilities made by chip producers as an additional tool for solving specific problems. This approach will decrease the time needed to learn CAD systems and will allow to focus on studying methods of analysis and design of analogue circuits using these CAD systems.

\section{The concept of use of CAD systems in all kinds of learning activities}

The curriculum of engineering courses, which include the analogue circuitry, usually has the following types of classroom activities: lectures, seminars, laboratory practicals. The classical approach includes learning the basics of theory in the lectures, learning the operation of basic circuits and solving basic problems without using any computing technologies in the seminars, and getting skills of working with circuits in the laboratory practicals (figure 1). This approach is not relevant anymore, because the students do not acquire skills of working with modern CAD systems, therefore they do not meet the requirements of the labour market.

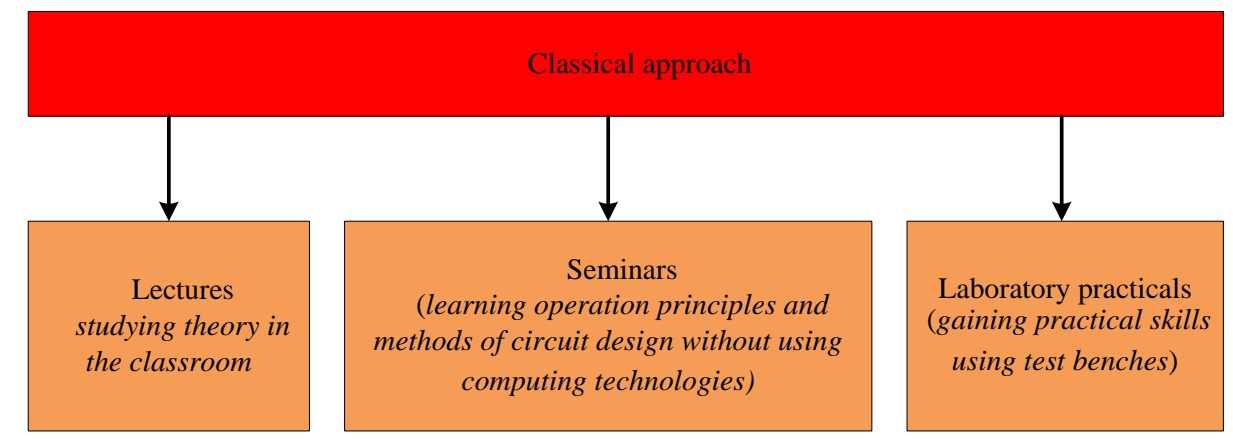

Fig. 1. Classical approach to the distribution of learning material in the curriculum.

The alternative to the classical approach is learning to use CAD systems in laboratory practicals, while completely excluding the use of laboratory test benches (figure 2). This approach originates from the 1990s and seems to be a consequence of economic conditions, when it was impossible to create new and support existing laboratory test benches. This substitution is unacceptable for engineering courses, because that makes the specialist lack the skills of working with real circuit technology, and his competences are limited to only working with models of analogue devices. 


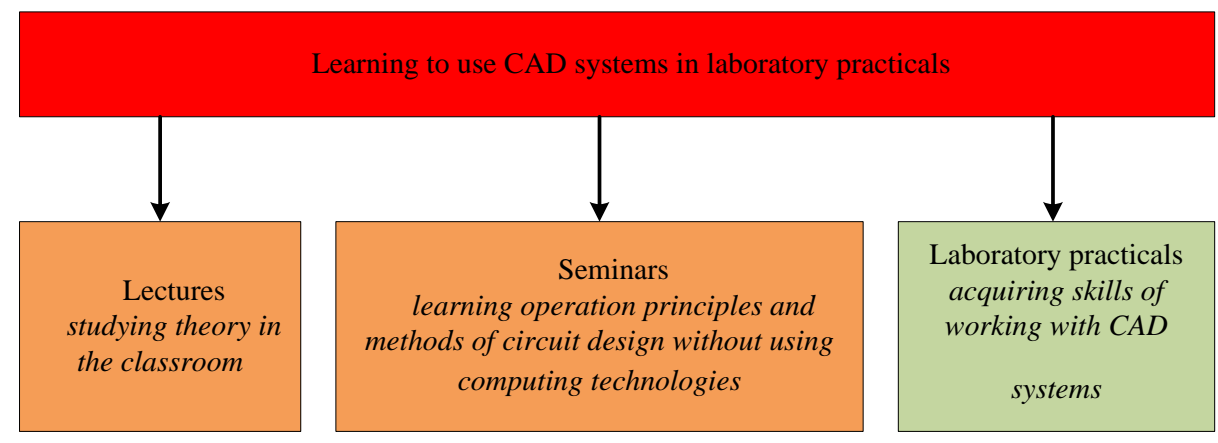

Fig. 2. Alternative approach to the distribution of learning material in the curriculum.

The authors suggest the following approach of using CAD systems in studying of analogue circuitry (figure 3), where CAD systems have a function for each kind of classroom activities (table 1 ).

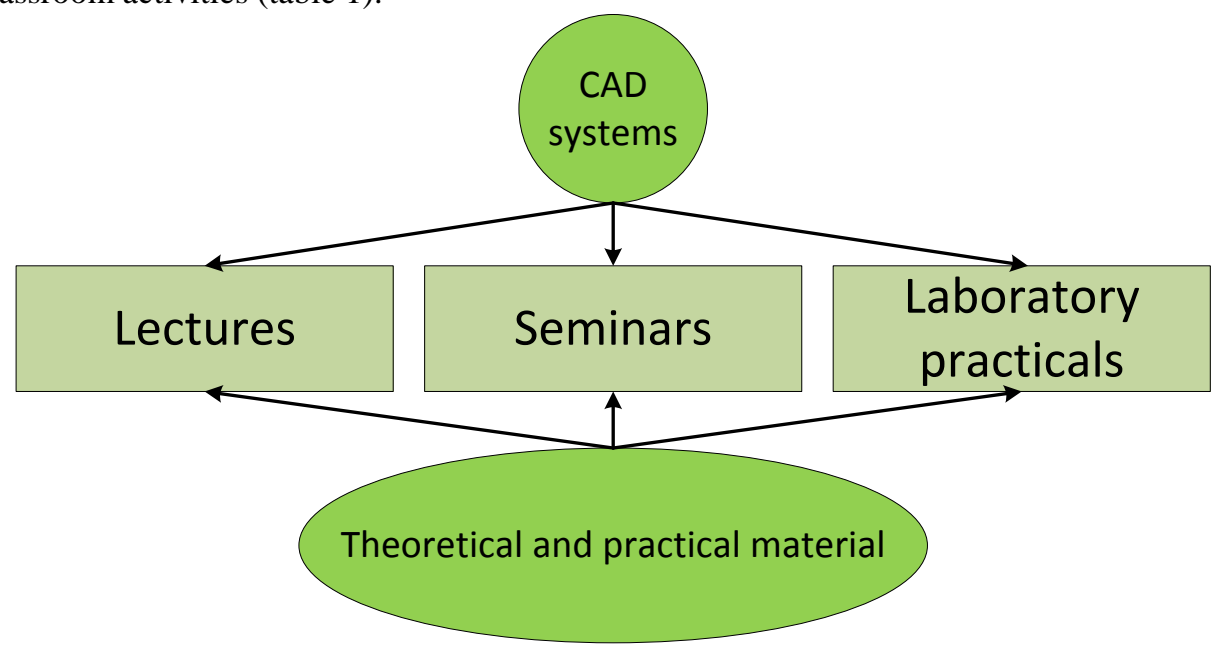

Fig. 3. Suggested approach of using CAD systems in training of the specialists in analogue circuitry.

In the lectures, CAD systems are the additional source of illustration of basic parameters of analogue elements. Therefore, CAD systems have the secondary function and help to show the main properties of analogue devices.

CAD systems play the key role in the seminars, where they allow to use new methods of circuit design for the already known circuits. In these classes it is possible not only to design a circuit, but also to get its temporal or frequency characteristics, calculate the operating point, compare different solutions, etc. 
Table 1. CAD system functions by the kind of classroom activities.

\begin{tabular}{|c|c|c|}
\hline $\begin{array}{c}\text { Classroom } \\
\text { activity }\end{array}$ & CAD system function & CAD system application example \\
\hline Lecture & $\begin{array}{c}\text { Visualization of lecture } \\
\text { material }\end{array}$ & $\begin{array}{c}\text { Visualization of the dependency of operational } \\
\text { amplifier parameters on the temperature }\end{array}$ \\
\hline Seminar & $\begin{array}{c}\text { New methods of } \\
\text { computation and chip } \\
\text { analysis }\end{array}$ & $\begin{array}{c}\text { Estimate of sensitivity of amplification factor } \\
\text { of the circuit to the precision of the elements } \\
\text { using Monte-Carlo method }\end{array}$ \\
\hline $\begin{array}{c}\text { Laboratory } \\
\text { practicals }\end{array}$ & $\begin{array}{c}\text { Verification of } \\
\text { laboratory test bench } \\
\text { data }\end{array}$ & $\begin{array}{c}\text { Comparison of the temporal data realization in } \\
\text { the simulation and on the oscillograph screen }\end{array}$ \\
\hline
\end{tabular}

In the laboratory practicals, CAD systems are used as a tool for finding errors while interacting with the test benches. Students are asked to design the same circuit both in the CAD system and on the test bench. If there is a significant difference in the results, it may indicate an error, and it may help to find these errors.

The experience in teaching the basics of analogue circuitry in the Autonomous Information and Control Systems department of Bauman Moscow State Technical University using the suggested approach has shown positive results in the acquisition of new competencies by the students. Moreover, positive feedback on the acquired knowledge and skills of students has also been received from the enterprises where students had the internship.

\section{Example of using CAD systems in learning active filters on operational amplifiers}

Despite the development of digital signal processing, which also includes digital filtering, analogue filters are an integral part of electronic circuits. They provide the matching of inputs of the analogue-digital converter with the signal sources. Moreover, the devices which only provide the analogue processing are still relevant.

To ensure full coverage of this issue, including both theoretical and practical aspects, we suggest the following plan for the course.

In the lectures, we consider analogue filters using the filter theory applied to operational amplifiers [6]. We consider only frequency-selective filters, which include low-pass filters, high-pass filters, band-pass filters and stop-band filters. An ideal filter assumes no transition band between passband and stopband; however, it is impossible to realize. Because of that, in practice we use filters with a transfer function represented as a ratio of two polynomials [8]:

$$
K(s)=\frac{A(s)}{B(s)}=\frac{a_{m} s^{m}+a_{m-1} s^{m-1}+\ldots+a_{1} s^{1}+a_{0}}{b_{n} s^{n}+b_{n-1} s^{n-1}+\ldots+b_{1} s^{1}+b_{0}},
$$

where $A(s)$ is a numerator polynomial, $B(s)$ is a denominator polynomial, $a_{i}, b_{i}$ are real constant coefficients, $m, n=1,2,3 \ldots(m \leq n)$.

The higher the order of a filter, the closer its characteristics are to the ideal filter. However, it is impossible to realize a filter of order higher than 2 on one operational amplifier. Because of that, we focus on filters of the $1^{\text {st }}$ and $2^{\text {nd }}$ order. Since the principle of 
filter circuit design does not depend on the passband, we consider only low-pass filters. A transfer function of a low-pass filter of the $1^{\text {st }}$ order has a following form:

$$
K(s)=\frac{K_{0} \omega_{0}}{s+\omega_{0}},
$$

where $K_{0}$ is an amplification factor in the passband, $\omega_{0}$ is a pole frequency, which in this case coincides with a cut-off frequency.

This filter is realized based on the operation amplifier using an inverting integrator circuit, with the cut-off frequency and amplification factor determined using resistors and capacitors. A transfer function of a low-pass filter of the $2^{\text {nd }}$ order has a following form:

$$
K(s)=\frac{K_{0} \omega_{0}^{2}}{s^{2}+\frac{\omega_{0}}{Q} s+\omega_{0}^{2}},
$$

where $K_{0}$ is an amplification factor in the passband, $\omega_{0}$ is a pole frequency, $Q$ is a filter quality.

There are several ways of circuit implementation of this transfer function, with the most popular being Sallen-Key filter and a filter with multiple-loop feedback.

Sallen-Key topology, also known as voltage-controlled voltage-source filter topology, has been first introduced in 1955 by R. P. Sallen and E. L. Key of Lincoln Laboratory of Massachusetts Institute of Technology[21]. One of the reasons for its popularity is the low dependency of filter characteristics on the operational amplifier characteristics. This is because the operational amplifier works as an amplifier, not an integrator, that reduces the bandwidth. Therefore, for a given operation amplifier it is possible to realize a filter of a higher frequency compared to other topologies. Another advantage of Sallen-Key topology is a low range of nominals of passive elements (resistors and capacitors), which is good in technological terms. A disadvantage is a high sensitivity of a filter quality to the change of nominals of the elements.

A topology with a multiple-loop feedback uses an operational amplifier in the integrator mode. Therefore, there is a higher dependency of a transfer function on the parameters of the operational amplifier, than in the Sallen-Key realization. Because of that, it is harder to realize higher values of filter quality, especially on higher frequencies. Moreover, the range of nominals of elements is higher compared to the Sallen-Key structure. However, the sensitivity of a filter quality to the change of nominals of elements is lower.

CAD systems are used to show the changes of filter characteristics depending on the chosen structure and operational amplifier. For example, the use of variation of nominals of filter elements using Monte-Carlo method allows to visually estimate the change of filter quality depending on the chosen structure.

In the seminars, we consider the development of filter circuit under the given frequency restraints. Generally, there are the following stages:

determine the required mathematical transfer function;

represent the transfer function as a product of the $1^{\text {st }}$ and $2^{\text {nd }}$ order polynomials;

choose the structure of an analogue filter on operational amplifier and implement the required filter as a cascade of filters of the $1^{\text {st }}$ and $2^{\text {nd }}$ order.

There are several ways to obtain a transfer function under the given frequency restraints, having a different behaviour in the terms of amplitude and phase characteristics. The choice 
of a specific method is based on the requirements to the system in the terms of the signal processing (in the frequency or time domain), number of used elements, etc. In practice, the following types of filters are usually used: Butterworth filter, Chebyshev filter, Bessel filter.

Butterworth filter is the best compromise between the fastest transition from the passband to the stopband and the linearity of the phase characteristic. Normalized poles of Butterworth filter are located on the unit circle (in the s-plane) and are given by the following expression:

$$
s_{k}=-\sin \left(\frac{(2 k-1) \pi}{2 n}\right)+\mathrm{j} \cos \left(\frac{(2 k-1) \pi}{2 n}\right),
$$

where $k$ is a pole pair number, and $n$ is a number of poles.

Chebyshev filter has a smaller transition region than a Butterworth filter of the same order, achieved by the non-monotonicity of the amplitude characteristic in the passband. In order to do that, the Chebyshev filter poles are formed by shifting Butterworth filter poles to the right, forming an ellipse. It is done by multiplying the real part of the pole on the $K_{R}$ coefficient, and the imaginary part on the $K_{I}$ coefficient, defined as

$$
\begin{aligned}
& K_{R}=\operatorname{sh}\left(\frac{1}{n} \operatorname{arsh}\left(\frac{1}{\varepsilon}\right)\right) ; \\
& K_{I}=\operatorname{ch}\left(\frac{1}{n} \operatorname{arsh}\left(\frac{1}{\varepsilon}\right)\right),
\end{aligned}
$$

where $n$ is a filter order, $\varepsilon=\left(10^{R_{d B} / 10}-1\right)^{1 / 2}$, and $R_{d B}$ are ripples of the amplitude characteristic in the passband.

The Bessel filter is optimized for getting smooth impulse response because of the linear phase in the passband. However, the transition from the passband and stopband is slower than in Butterworth and Chebyshev filters. It is preferred when the post-processing is in the time domain.

The poles of Bessel filters can be determined by placing the poles on the circle so that the distance between their imaginary parts is

$$
\frac{2}{n}
$$

where $n$ is a number of poles.

Mathematical tools used in these stages are well-known, and they are described in different sources [6-9], so the students can learn them on their own if necessary. There is practical interest in gaining skills of working with software for filter synthesis and comparison of the properties of the filters. The FilterPro utility by Texas Instruments [14] can be used for these purposes. The result for a low frequency filter of the $4^{\text {th }}$ order, implemented as a Chebyshev filter with multiple-loop feedback is shown on the figure 4. 


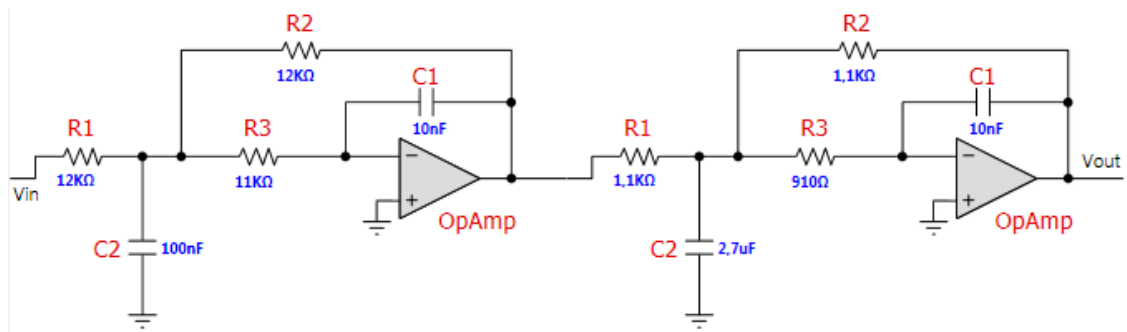

Fig. 4. The output of FilterPro utility by Texas Instruments.

The advantage of this utility is the ability to choose from the series of preferred numbers of passive elements for circuit implementation, and to display the frequency characteristics of the circuit, while taking into account the calculated and rounded values. Therefore, the student acquires the skill of working with modern software for solving a particular engineering problem and can focus on comparing different ways of filter design under the same frequency constraint.

In the laboratory practicals, the students study different structures of filters of $1^{\text {st }}$ and $2^{\text {nd }}$ order. The ASLK PRO circuit by MIKROE company is used as a test bench. MicroCap CAD system is used here only as a source of reference data, which is compared with the results from the test bench. The students use the software by themselves, because they already gained the skills of using it in the seminars.

Therefore, by this example we show the use of CAD systems in all kinds of classroom activities in the engineering courses. This approach makes the students acquire both theoretical knowledge and practical skills of using modern software tools.

\section{Conclusion}

Currently, a specialist in the field of analogue circuitry has a wide range of tools that increase the efficiency of his work. A significant part of these tools are software products, which reduce the routine of creating the electronic devices, and provide a high-quality visualization of main operating characteristics of these devices. Therefore, in the process of learning, the student should extensively study CAD systems, but that should not affect the practical skills acquired on the laboratory practicals. The suggested approach of use of CAD systems in all kinds of classroom activities allows to acquire skills of rationally using software when solving theoretical and practical problems.

\section{References}

1. V. Artuhov, O. Brytov, FIR Filter Design by Micro-Cap Tools, 2019 IEEE 39th International Conference on Electronics and Nanotechnology (ELNANO), pp. 626629), IEEE, April (2019)

2. A.V. Boldyrev, Simulation of the pulse voltage converter power part, 2017 International Conference on Industrial Engineering, Applications and Manufacturing (ICIEAM), pp. 1-4, IEEE, May (2017)

3. M.A. Amelina, S.A. Amelin, Programma skhemotekhnicheskogo modelirovaniya Micro Cap 8 [Circuit simulation software Micro Cap 8], Moskow, Goryachaya liniyaTelekom (2017)

4. J.M. Fiore, Operational amplifiers and linear integrated circuits: Theory and application, West Publishing Company (1992) 
5. A.N. Borodjieva \& A.V. Manukova-Marinova, Analysis and design of active filters with generalized impedance converter, 27th International Spring Seminar on Electronics Technology: Meeting the Challenges of Electronics Technology Progress, 2004, Vol. 3, pp. 398-404,IEEE, May (2004)

6. R. Schaumann, Mac Van Valkenburg, Design of analog filters (2001)

7. L.P. Huelsman, Active and passive analog filter design: an introduction, McGraw-Hill (1993)

8. R. Schaumman, M.S. Ghausi \& K.R. Laker, Design of analog filters: passive, active $R C$ and switched capacitors. London, UK: Prentice Hall, section, 7(1), 429 (1990)

9. P.L. Taylor, Flexible design method for active RC two-ports, Proceedings of the Institution of Electrical Engineers, Vol. 110, No. 9, pp. 1607-1616, IET Digital Library, September (1963)

10. S. Meshkov, M. Makeev, V. Shashurin, Y. Tsvetkov \& B. Khlopov, Microelectronics Devices Optimal Design Methodology with Regard to Technological and Operation Factors, Energy Management of Municipal Transportation Facilities and Transport, December 2018, pp. 517-523, Springer, Cham (2018)

11. A. Krasovsky, S. Vasyukov \& I. Murzin, Electrical Model of Car Power Wiring for Transmission of Pulse Control Signals Through it, 2019 International Russian Automation Conference (RusAutoCon), September 2019, pp. 1-5, IEEE (2019)

12. M. Brinson \& V. Kuznetsov, Current conveyor macromodels for wideband RF circuit design, 2016 MIXDES-23rd International Conference Mixed Design of Integrated Circuits and Systems, June 2016, pp. 62-67, IEEE (2016)

13. V.A. Shakhnov, L.A. Zinchenko \& E.V. Rezchikova, Modeling and simulation of nanoelectronics devices in cognitive nanoinformatics, International Conference on Micro-and Nano-Electronics 2014, Vol. 9440, p. 944018, December 2014, International Society for Optics and Photonics (2014)

14. B. Carter \& R. Mancini, Op Amps for everyone, Newnes (2017)

15. S. Juneja, R. Sharma \& A. Mantri, Strategic Partnership with Industry to Strengthen Core Competency in Electronics-Need, Opportunities, Challenges and Success Stories, 2017 7th World Engineering Education Forum (WEEF), November 2017, pp. 562-567, IEEE (2017)

16. D. Belega, R. Pazsitka \& D. Stoiciu, Measurement experiments performed using the ASLK PRO board, 2016 12th IEEE International Symposium on Electronics and Telecommunications (ISETC), October 2016, pp. 162-166, IEEE (2016)

17. Y. Vagapov, A. Anuchin \& M. Pushchin, Modelling of a photovoltaic array using Analog System Lab Kit Pro board, 2018 IEEE Conference of Russian Young Researchers in Electrical and Electronic Engineering (EIConRus), March 2018, pp. 1791-1794, IEEE (2018)

18. A.G. Kimura, K.W. Liu, S. Prabhu, S.B. Bibyk \& G. Creech, Trusted verification test bench development for phase-locked loop (PLL) hardware insertion, 2013 IEEE 56th International Midwest Symposium on Circuits and Systems (MWSCAS), August 2013, pp. 1208-1211, IEEE (2013)

19. J. Barker, J. Cline, K. Sheng \& S. Bibyk, Electronic system hardware exploration in an inverted classroom, 2013 IEEE 56th International Midwest Symposium on Circuits and Systems (MWSCAS), August 2013, pp. 578-581, IEEE (2013) 
20. A.K. Gond, R. Gupta, S. Basu, S. Pandit \& S. Barman, Behavioral Modeling of Differential Inductive Seismic Sensor and Implementation of Its Readout Circuit, Communication, Devices, and Computing, pp. 253-262, Springer, Singapore (2017)

21. R.P. Sallen \& E.L. Key, A practical method of designing $R C$ active filters, IRE Transactions on Circuit Theory, 2(1), pp. 74-85 (1995) 